\title{
Commentary
}

\section{How a Network Came Together to Manage a Large Research Program During the Pandemic}

\author{
Tamara Friedman, PhD'*; Elli G. Paleoudis, MS, PhD';Avery Freed, CIP'; Susan Adler, MPA'; Cheryl Fittizzi, MBA, RN, CIP'; \\ Ihor Sawczuk, MD, FACS ${ }^{1,2}$
}

'Office of Research Administration, Hackensack Meridian Health, 40 Prospect St, Hackensack, NJ 0760 I, USA

${ }^{2}$ Hackensack Meridian Health School of Medicine, 340 Kingsland St Ste 123, Nutley, NJ 07I I 0, USA

\section{"Corresponding author}

Tamara Friedman, PhD

Manager, Research Communications and Education, Office of Research Administration, Hackensack University Medical Center, 40 Prospect St, Second Floor, Hackensack, NJ 0760I, USA;Tel. 55I-996-2193; E-mail: tamara.friedman@hackensackmeridian.org

\section{Article information}

Received: June II ${ }^{\text {th }}$, 2020; Revised: July $17^{\text {th }}$, 2020; Accepted: July I7 $7^{\text {th }}, 2020$; Published: July $27^{\text {th }}, 2020$

Cite this article

Friedman T, Paleoudis EG, Freed A, Adler S, Fittizzi C, Sawczuk I. How a network came together to manage a large research program during the pandemic. Clin Trial Pract Open J. 2020; 3(I): 20-22. doi: 10.17I40/CTPOJ-3-II4

\section{ABSTRACT}

Hackensack Meridian Health, a large New Jersey network comprised of 17 hospitals, a medical school, and 200 ambulatory care centers, executes a large research program with approximately 1000 active human subjects studies. With the arrival of the coronavirus disease-2019 (COVID-19) pandemic, the Office of Research Administration was confronted with a series of challenges. These included minimizing face-to-face contact among research participants and staff, allocating limited resources to handle the influx of COVID-19 related proposals, providing human subjects protection training to the physicians and staff who were needed to run the clinical trials, adjusting bio-repository activities to meet the increased demand for samples, and disseminating all of the new regulatory and operational information to the thousands of researchers in the network. The network's success in modifying its research program quickly and effectively to adapt to the changing landscape can be attributed, in large part, to the centralized nature of the research administration.

\section{Keywords}

Research administration; COVID-19; Network.

\section{INTRODUCTION}

$\mathrm{H}$ ackensack Meridian Health (HMH) is a network of 17 hospitals, a medical school, and over 200 ambulatory centers with a centralized research administration. The Office of Research Administration (ORA) supports all clinical and basic research across $\mathrm{HMH}$ and within the last year oversaw approximately 900-1000 active human subjects research protocols, including sponsored and investigator initiated studies.

The HMH network is located in New Jersey, where coronavirus disease-2019's (COVID-19's) impact has been considerable; our state is one of the country's hardest hit. At this time, there have been thousands of COVID-related hospitalizations in the state and over 12,000 COVID-related deaths. ${ }^{1}$ The rapidity with which hospital and research operations were affected by the pandemic propelled the ORA to quickly adapt and adopt certain strategies and new institutional policies. The main challenges encoun- tered and the corresponding responses from the ORA are outlined below.

\section{Challenges and Responses}

One of our first concerns was ensuring that any unnecessary faceto-face contact among research participants and research staff was minimized. The research administration accomplished this by following federal guidelines ${ }^{2}$ and suspending all clinical research that was not COVID-related and that didn't have the potential for direct benefit for the participants. Researchers were also urged to utilize creative approaches in lieu of face-to-face contact. For example, they were coached to obtain consent over the phone and to utilize DocuSign $^{\mathcal{C}}, 3$ a secure means of obtaining electronic signatures for the consent forms. These alternative approaches allowed the institution to remain compliant and to promote patient autonomy. ${ }^{4}$ 
There was also the challenge of developing a strategy to manage the incoming studies; the ORA anticipated a significant influx of COVID-related proposals for Institutional Review Board (IRB) review. An action plan was created to limit duplicate efforts across the network, to best utilize available resources, and to ensure that the IRB members and staff were able to facilitate time sensitive reviews. This was accomplished by establishing a Steering Committee through which the COVID-related proposals would be pre-reviewed prior to proceeding to the IRB. This pre-review considered duplicative efforts, scientific merit, feasibility, and regulatory compliance prior to approval. Ultimately, 227 proposals were submitted to the Committee and fewer than $40 \%$ were approved for submission to the IRB. In addition to the Steering Committee, an external IRB was utilized for some of the sponsored studies. Finally, research staff from various departments were recruited to provide assistance in handling the COVID-19 research operations. This brought researchers and staff from different departments together when their paths might not have otherwise crossed.

Another immediate challenge that presented itself was related to the sudden involvement in research of certain physicians who had never engaged in research before. There was also an urgent need for assistance with data entry, so many staff members who had never served on research teams were recruited to that end. It became necessary to consider the balance between the demands for people to support clinical trials that could be life-saving with the fundamental requirement of ensuring that the participants were adequately protected. Researchers still needed to be trained in human subjects protection and relevant regulations, and conflicts of interest needed to be checked. To address this, an abridged training that condensed the most relevant concepts was provided to the researchers whose participation in the studies was imminently needed, but did not have time to complete the standard training. This was on the condition that they committed to complete the full Collaborative Institutional Training Initiative (CITI) training within 60-days. Additionally, a streamlined approach to the disclosure/clearance of conflicts of interest was developed that did not require electronic registration and form completion.

Like other institutions, HMH experienced a critical need for COVID-19 samples and data to study the virus in vitro. ${ }^{5}$ The network bio-repository responded by ramping up its efforts, including collecting additional samples during standard of care, discards from pathology, and also from deceased patients. They were able to increase their sample collection by over $400 \%$ over the course of three weeks from the pandemic's onset.

Finally, from its beginning and as the pandemic evolved, it has been necessary to disseminate information quickly and efficiently to everyone involved in research, including researchers, staff, and IRB members. A communications team was established and charged with coordinating the dissemination of guidance and institutional policies. The team instituted a research hotline with $24 / 7$ coverage, shared daily research updates with thousands of researchers, and delivered a series of presentations to the research community.

\section{DISCUSSION}

The ORA and network researchers came together and were overall successful in their efforts; however, as expected, there were some limitations that should be considered for future crises. First, while the Steering Committee was helpful in allocating limited resources and ensuring stronger studies progressed for IRB submission, the pre-review did contribute to some delays in the projects moving through the review process. Second, following-up to ensure that all of the researchers who completed the abridged training ultimately completed the full training within 60-days became cumbersome; it would have been better to have created a more streamlined approach. Third, while the bio-repository was incredibly prolific, it was initially limited by a staffing shortage, which took some time to rectify. Finally, while the presentations were well-attended, some misunderstandings persisted. This may be attributed to people not having the time to read/remember all of the disseminated information.

This experience has demonstrated the importance of maintaining a centralized research administration. Having one unified group of people making key decisions regarding the research program and conveying them to the research community allowed for the research to progress successfully in the face of COVID-19. Other factors that were important to the research program management included our efforts to coordinate groups within the research administration effectively, not work in silos, and to share information regularly throughout the network. ${ }^{6}$ As a result, $\mathrm{HMH}$ researchers have published extensively, and $\mathrm{HMH}$ has served as a site for numerous COVID-19 sponsored trials that have offered treatment alternatives to patients.

\section{ACKNOWLEDGEMENTS}

We would like to acknowledge the hard work of Daniel Alderson, Yael Kramer, Tracy Micalizzi, David Candelmo, and Sergio Garcia whose dedication contributed to the success of our research program during the pandemic.

\section{AUTHORS' CONTRIBUTIONS}

Dr. Friedman was the primary writer. Dr. Gourna Paleoudis, Ms. Freed, and Ms. Adler edited the manuscript. Ms. Fittizzi reviewed the manuscipt. She and Dr. Sawczuk oversee the research administration, about which this article is written.

\section{CONFLICTS OF INTEREST}

The authors declare that they have no conflicts of interest.

\section{REFERENCES |}

1. Department of Health. New Jersey COVID-19 Dashboard. state.nj.us Web site. https://www.state.nj.us/health/cd/topics/ covid2019_dashboard.shtml. Accessed June 10, 2020. 
2. U.S. Food and Drug Administration (FDA) COVID-19-Related Guidance Documents for Industry, FDA Staff, and Other Stakeholders.fda.gov Web site. https://www.fda.gov/emergency-preparedness-and-response/coronavirus-disease-2019-covid-19/ covid-19-related-guidance-documents-industry-fda-staff-and-other-stakeholders. Accessed June 10, 2020.

3. DocuSign. No matter what, no matter where. docusign Web site. https://www.docusign.com/. Accessed June 10, 2020.

4. Cocanour CS. Informed consent-It's more than a signature on a piece of paper. Am J Surg. 2017; 214(6): 993-997. doi: 10.1016/j. amjsurg.2017.09.015

5. National Institutes of Health, All of Us Research Program launches COVID-19 research initiatives. nih.gov Web site. https:/ / www.nih.gov/news-events/news-releases/all-us-research-program-launches-covid-19-research-initiatives. Accessed June 10, 2020.

6. Thornton, K. An Analysis of Academic Research AdministrationCentral Office Structures. [master's thesis]. Baltimore, MD, USA: Johns Hopkins University; 2017. 